\title{
SUFFICIENT CONDITIONS FOR THE GENERAL PROBLEM OF MAYER WITH VARIABLE END POINTS*
}

\author{
BY \\ M. R. HESTENES
}

1. Introduction. The problem of the calculus of variations to be considered here is the general problem of Mayer with variable end points as proposed by Bliss (V, p. 305) $\dagger$ and recently studied for a particular case in a joint paper by Bliss and Hestenes (XVI). As was remarked in the latter paper the general problem of Mayer is equivalent to the problem of Bolza, but the sets of sufficient conditions which have been given by Morse and Bliss for the problem of Bolza are not applicable to the problem of Mayer without further modification. In view of this fact it is the purpose of the present paper to establish a set of sufficient conditions for the general problem of Mayer with variable end points. The proofs here given are equally applicable to the problem of Bolza considered as a problem of Mayer.

The procedure used is similar to that used by Bliss for the problem of Bolza (XII, pp. 261-274). We first derive in $\$ 4$ a further necessary condition analogous to that deduced by Bliss for the problem of Bolza. In $\$ 5$ we construct an auxiliary problem of Mayer of the type discussed by Bliss and Hestenes (XVI). Their results are then applied in $\$ \S 6$ and 8 to the general problem of Mayer by methods closely related to those suggested by Mayer (XIII, pp. 436-465) and Hahn (XIV, pp. 127-136).

2. Statement of the problem. In the following pages the notation and the terminology used by Bliss and Hestenes for a particular problem of Mayer will be used throughout (XVI, pp. 306-309). In addition it will be understood that the indices $\mu, \nu$ have the ranges

$$
\mu, \nu=1, \cdots, p<2 n+1 .
$$

The general problem of Mayer is then that of minimizing a function $g\left[x_{1}, y\left(x_{1}\right)\right.$, $\left.x_{2}, y\left(x_{2}\right)\right]$ in a class of arcs

$$
y_{i}=y_{i}(x)
$$$$
\left(x_{1} \leqq x \leqq x_{2}\right)
$$

which satisfy the differential equations and end conditions

$$
\phi_{\alpha}\left(x, y ; y^{\prime}\right)=0, \quad \psi_{\mu}\left[x_{1}, y\left(x_{1}\right), x_{2}, y\left(x_{2}\right)\right]=0 .
$$

* Presented to the Society, April 8, 1932; received by the editors June 9, 1932, and, revised, December 10, 1932.

$\dagger$ The Roman numerals in the parentheses in the text refer to the bibliographies at the end of the paper by Bliss and Hestenes, cited here as XVI, and at the end of the present paper. 
As before, the arcs (2:1) and the functions $\phi_{\alpha}, g, \psi_{\mu}$ will be assumed to have the continuity properties (a), (b), (c) (XVI, p. 306) in a neighborhood of a particular arc $E_{0}$ whose minimizing properties are to be studied, the determinant (2:1) appearing in (c) being now interpreted as a $(2 n+2) \times(p+1)$ dimensional matrix of rank $p+1$.

For the general problem of Mayer the first necessary condition as given by Bliss and Hestenes (XVI, p. 307) is modified as follows, and is readily established by the methods which they suggest. The theorem has also been established by Morse and Myers (X, p. 245).

I. ThE FIRST NECESSARY CONDITION. Every minimizing arc $E_{0}$ for the problem of Mayer with variable end points must satisfy, besides the conditions (XVI, p. 307)

$$
F_{y_{i^{\prime}}}=\int_{x_{1}}^{x} F_{y_{i}} d x+c_{i}, \quad \phi_{\alpha}=0,
$$

the further relation

$$
\left(F-y_{i}^{\prime} F_{y_{i^{\prime}}}\right) d x+\left.F_{y_{i^{\prime}}} d y_{i}\right|_{1} ^{2}+\lambda_{0} d g=0
$$

for every set of differentials $d x_{1}, d y_{i 1}, d x_{2}, d y_{i 2}$ satisfying the equations $d \psi_{\mu}=0$, $\lambda_{0}$ being a suitably chosen constant.

An admissible arc $E_{0}$ is said to be normal relative to the end conditions $\psi_{\mu}=0$ if there exist for it $p$ sets of admissible variations $\xi_{1}{ }^{\nu}, \xi_{2}{ }^{\nu}, \eta_{i}{ }^{\nu}(x)$ such that the determinant $\left|\Psi_{\mu}\left(\xi^{\nu}, \eta^{\nu}\right)\right|$ is different from zero (XVI, p. 307). For convenience an arc that is normal relative to the end conditions $\psi_{\mu}=0$ will be designated simply as normal.

THEOREM 2:1. An admissible arc that does not satisfy the necessary condition I is normal.

This follows at once because an admissible arc $E_{0}$ satisfies the necessary condition I if and only if every determinant of the form

$$
\left|\begin{array}{c}
G\left(\xi^{\sigma}, \eta^{\sigma}\right) \\
\Psi_{\mu}\left(\xi^{\sigma}, \eta^{\sigma}\right)
\end{array}\right| \quad(\sigma=1, \cdots, p+1)
$$

vanishes, where $\xi_{1}{ }^{\sigma}, \xi_{2}{ }^{\sigma}, \eta_{i}{ }^{\sigma}(x)$ are $p+1$ sets of admissible variations for $E_{0}$, and the function $G$ is obtained from $g$ in the same manner as $\Psi_{\mu}$ is obtained from $\psi_{\mu}$ (V, p. 309).

THEOREM 2:2. An admissible arc $E_{0}$ that satisfies the necessary condition I is normal if and only if there exist for it no set of multipliers $\lambda_{\alpha}(x)$, not vanishing 
simultaneously, with which it satisfies equations (2:2) and for which all $(p+1)$ rowed determinants of the matrix

$$
\left\|\begin{array}{cccc}
y_{i 1^{\prime}}^{\prime} F_{y_{i^{\prime}}}\left(x_{1}\right) & F_{y_{i^{\prime}}}\left(x_{1}\right) & y_{i 2}^{\prime} F_{y_{i}}\left(x_{2}\right) & -F_{y_{i^{\prime}}}\left(x_{2}\right) \\
\psi_{\mu x_{1}} & \psi_{\mu y_{i 1}} & \psi_{\mu x_{2}} & \psi_{\mu y_{i 2}}
\end{array}\right\|
$$

vanish. If $E_{0}$ is normal the constant $\lambda_{0}$ can be chosen to be unity, the multipliers $\lambda_{\alpha}(x)$ with which $E_{0}$ satisfies the conditions (2:2) and (2:3) being then unique.

This theorem is an obvious generalization of a theorem given by Bliss and Hestenes and can be proved by the same methods (XVI, p. 308). A similar theorem has been established by Bolza (III, p. 441).

3. Theorems on extremals. It is known that in the problems of Mayer a non-singular extremal arc can be imbedded in a $(2 n-1)$-parameter family of extremals (XVI, p. 311)

$$
y_{i}=y_{i}\left(x, c_{1}, \cdots, c_{2 n-1}\right), \lambda_{\alpha}=\lambda_{\alpha}\left(x, c_{1}, \cdots, c_{2 n-1}\right) \quad\left(x_{1} \leqq x \leqq x_{2}\right) .
$$

Further properties of this family are given in the following theorem:

THEOREM 3:1. Let $E_{0}$ be a member of the $(2 n-1)$-parameter family of extremals (3:1) for parameter values $\left(x_{10}, x_{20}, c_{0}\right)$. If the matrix

$$
\left\|\begin{array}{l}
y_{i c_{s}}\left(x_{1}, c\right) \\
y_{i c_{s}}\left(x_{2}, c\right)
\end{array}\right\|
$$

has rank $2 n-1$ on $E_{0}$, then there is a neighborhood $N$ of the ends of $E_{0}$ in $\left(x_{1} y_{1} x_{2} y_{2}\right)$-space such that the end values of every extremal of the family (3:1) with ends in $N$ satisfy a relation $W\left(x_{1}, y_{1}, x_{2}, y_{2}\right)=0$. Conversely, every pair of points $\left(x_{1}, y_{1}\right),\left(x_{2}, y_{2}\right)$ in $N$ satisfying the condition $W=0$ can be joined by an extremal $E$ of the family (3:1), and by taking $N$ sufficiently small the parameters $\left(x_{1}, x_{2}, c\right)$ belonging to $E$ will lie in a preassigned $\epsilon$-neighborhood of those belonging to $E_{0}$. The function $W$ has continuous partial derivatives of the first two orders in $N$.

The theorem can be proved as follows. Select $2 n$ constants $a_{i}, b_{i}$ such that the determinant

$$
\left|\begin{array}{ll}
y_{i c_{8}}\left(x_{1}, c\right) & a_{i} \\
y_{i c_{s}}\left(x_{2}, c\right) & b_{i}
\end{array}\right|
$$

is different from zero on $E_{0}$. Consider now the equations

$$
\begin{aligned}
& y_{i 1}=y_{i}\left(x_{1}, c\right)+W a_{i}, \\
& y_{i 2}=y_{i}\left(x_{2}, c\right)+W b_{i} .
\end{aligned}
$$

These equations are satisfied by the set $\left(x_{10}, y_{10}, x_{20}, y_{20}, c_{0}, W=0\right)$ belonging to $E_{0}$. Furthermore the functional determinant with respect to the variables $c_{s}, W$ is the determinant $(3: 3)$ and is therefore different from zero on $E_{0}$. It 
follows that equations $(3: 4)$ have a unique solution

$$
c_{s}=c_{s}\left(x_{1}, y_{1}, x_{2}, y_{2}\right), \quad W=W\left(x_{1}, y_{1}, x_{2}, y_{2}\right)
$$

in a neighborhood $N$ of the end values $\left(x_{10}, y_{10}, x_{20}, y_{20}\right)$ belonging to $E_{0}$. The right members of equations $(3: 5)$ have continuous first and second derivatives in $N$ since the right and left members of equations (3:4) have such derivatives. If now the end values of an extremal are in $N$, then these end values must satisfy the relation $W\left(x_{1}, y_{1}, x_{2}, y_{2}\right)=0$ since the solutions of equations (3:4) are unique. Furthermore every set of values $\left(x_{1}, y_{1}, x_{2}, y_{2}\right)$ in $N$ satisfying the relation $W=0$ are the end values of an extremal $E$ with parameter válues $\left[x_{1}, x_{2}, c\left(x_{1}, y_{1}, x_{2}, y_{2}\right)\right]$, and by taking $N$ sufficiently small these parameter values will lie in a preassigned $\epsilon$-neighborhood of those belonging to $E_{0}$. Hence the theorem is proved.

It is now possible to give an interesting geometric interpretation of normality.

THEOREM 3:2. A non-singular extremal arc $E_{0}$ whose matrix $(3: 2)$ has rank $2 n-1$ is normal if and only if in the space of points $\left(x_{1}, y_{1}, x_{2}, y_{2}\right)$ the extremal end manifold $W=0$ and the terminal manifold $\psi_{\mu}=0$ are not tangent to each other at the point $\left(x_{10}, y_{10}, x_{20}, y_{20}\right)$ defining the end values of $E_{0}$.

To prove this it is sufficient, as is readily seen, to show that the derivatives $W_{x_{1}}, W_{y_{i 1}}, W_{x_{2}}, W_{y_{i 2}}$ are proportional to the elements of the first row of the matrix $(2: 4)$. These derivatives have this property because the relation $F_{y_{i}} \eta_{i}$ $=$ constant along extremals (XVI, p. 307) with $\eta_{i}=y_{i c_{s}} d c_{s}$ implies that the differentials $d x_{1}, d y_{i 1}, d x_{2}, d y_{i 2}, d c_{s}, d W$ belonging to equations (3:4) satisfy the relation

$$
\left.F_{y^{\prime}}\left(d y_{i}-y_{i}^{\prime} d x\right)\right|_{1} ^{2}=\left.F_{y^{\prime}} y_{i c_{s}} d c_{s}\right|_{1} ^{2}+h d W=h d W
$$

where $h=b_{i} F_{y_{i^{\prime}}}\left(x_{2}\right)-a_{i} F_{y_{i^{\prime}}}\left(x_{1}\right)$. If $h=0$ on $E_{0}$ then on account of the relation $F_{y_{i}{ }^{\prime}} \eta_{i}=$ constant, the determinant (3:3) would vanish on $E_{0}$ which is not the case. Hence $h \neq 0$ on $E_{0}$ and

$$
\begin{aligned}
y_{i 1}^{\prime} F_{y_{i^{\prime}}}\left(x_{1}\right) & =h W_{x_{1}}, \quad-F_{y_{i^{\prime}}}\left(x_{1}\right)=h W_{y_{i 1}}, \\
-y_{i 2}^{\prime} F_{y_{i^{\prime}}}\left(x_{2}\right) & =h W_{x_{2}}, \quad F_{y_{i^{\prime}}}\left(x_{2}\right)=h W_{y_{i 2}}
\end{aligned}
$$

as was to be proved.

4. The necessary condition of Mayer. The necessary condition of Mayer for the problem of Bolza, as stated by Bliss (XII, p. 266), is also valid for the problem of Mayer considered here.* In order to derive this condition we sup-

* The proof is somewhat different from that given by Bliss for the problem of Bolza. He has called my attention to the fact that the argument which he used is inadequate in the case when the ends of $E_{0}$ are conjugate, and has suggested the modifications indicated here. 
pose that $E_{0}$ is a normal non-singular minimizing arc without corners and hence an extremal arc. If $\xi_{1}, \xi_{2}, \eta_{i}(x)$ are a set of admissible variations for $E_{0}$ which satisfy the conditions $\Psi_{\mu}(\xi, \eta)=0$, then $E_{0}$ is a member of a oneparameter family of admissible arcs with ends satisfying the conditions $\psi_{\mu}=0$ and having $\xi_{1}, \xi_{2}, \eta_{i}(x)$ as its variations along $E_{0}$ (IX, p. 695). For such a family the second variation of the function $g$ to be minimized is expressible along $E_{0}$ in the form

$$
I_{2}=\left(F_{x}+y_{i}^{\prime} F_{y_{i}}\right) \xi^{2}+\left.2 F_{y_{i}} \eta_{i} \xi\right|_{1} ^{2}+2\left(Q+l_{\mu} Q_{\mu}\right)+\int_{x_{1}}^{x_{2}} 2 \omega\left(x, \eta, \eta^{\prime}\right) d x
$$

where $Q, Q_{\mu}$ are quadratic forms in $\xi_{1}, \eta_{i}\left(x_{1}\right), \xi_{2}, \eta_{i}\left(x_{2}\right)$ whose coefficients are the second derivatives of the functions $g, \psi_{\mu}$, respectively, and

$$
2 \omega\left(x, \eta, \eta^{\prime}\right)=F_{y_{i} y_{k}} \eta_{i} \eta_{k}+2 F_{y_{i} y_{k^{\prime}} \eta_{i} \eta_{k}^{\prime}}+F_{y_{i^{\prime}} y_{k^{\prime}} \eta_{i}^{\prime} \eta_{k}^{\prime}} .
$$

This form for $I_{2}$ is readily obtained with the help of the transversality condition (2:3) by the methods used by Bliss and Hestenes (XVI, pp. 311-312). Let us consider variations satisfying the equations $\Psi_{\mu}(\xi, \eta)=0$ along $E_{0}$, and of the special form $\xi_{1}=d x_{1}, \xi_{2}=d x_{2}, \eta_{i}=\delta y_{i}=y_{i c_{s}} d c_{s}$, where the functions $y_{i}(x, c)$ are those defining the $(2 n-1)$-parameter family $(3: 1)$ of extremals to which $E_{0}$ belongs. For such variations the second variation $(4: 1)$ can also be expressed in the form

$$
\begin{aligned}
d^{2} g= & \left(F_{x}+y_{i}^{\prime} F_{y_{i}}\right) d x^{2}+2 F_{y_{i}} \delta y_{i} d x \\
& +\left.\delta y_{i} \Omega_{\eta_{i^{\prime}}}\left(x, \delta y, \delta y^{\prime}, \delta \lambda\right)\right|_{1} ^{2}+2\left(Q+l_{\mu} Q_{\mu}\right)
\end{aligned}
$$

given by Bliss (XII, p. 266), where $\delta \lambda_{\alpha}=\lambda_{\alpha c_{s}} d c_{s}$ and

$$
\Omega\left(x, \eta, \eta^{\prime}, \mu\right)=\omega\left(x, \eta, \eta^{\prime}\right)+\mu_{\alpha}\left(\phi_{\alpha y_{i}} \eta_{i}+\phi_{\alpha y_{i}} \eta_{i}^{\prime}\right) .
$$

Since $E_{0}$ is a minimizing arc the expression $(4: 1)$ must be $\geqq 0$ for all sets of admissible variations $\xi_{1}, \xi_{2}, \eta_{i}(x)$ which satisfy the conditions $\Psi_{\mu}(\xi, \eta)=0$. In particular it must be $\geqq 0$ for variations $\xi_{1}=d x_{1}, \xi_{2}=d x_{2}, \eta_{i}=\delta y_{i}$ of the special type considered above satisfying the conditions $d \psi_{\mu} \equiv \Psi_{\mu}(d x, \delta y)=0$. We have therefore the following result:

IV. THE NECESSARY CONDITION OF MAYER. For a normal non-singular minimizing arc $E_{0}$ without corners the quadratic form (4:2) must satisfy the condition $d^{2} g \geqq 0$ for all sets $\left(d x_{1}, d x_{2}, d c_{s}\right) \neq(0,0,0)$ which satisfy the equations $d \psi_{\mu}=0$. Furthermore between the end points 1 and 2 on $E_{0}$ there can be no point 3 conjugate to 1 defined by a value $x_{3}$ such that $E_{0}$ is normal on the interval $x_{3} x_{2}$. 
The last statement is a slight modification of the condition IV deduced by Bliss and Hestenes for problems of Mayer having $2 n+1$ end conditions (XVI, p. 315), valid here for $E_{0}$ since $E_{0}$ must also be a minimizing arc for such a problem, as will be seen in the next section.

5. An auxiliary problem of Mayer. In order to construct a problem of Mayer of the type described in the last paragraph we suppose that $E_{0}$ is a minimizing arc for the general problem of Mayer considered here. Its end values $\left(x_{10}, y_{10}, x_{20}, y_{20}\right)$ satisfy the conditions $\psi_{\mu}=0(\mu=1, \cdots, p)$. Adjoin to the functions $\psi_{\mu}, 2 n+1-p$ functions $\psi_{\tau}\left(x_{1}, y_{1}, x_{2}, y_{2}\right)(\tau=p+1, \cdots$, $2 n+1)$ possessing continuous first and second partial derivatives in a neighborhood of the values $\left(x_{10}, y_{10}, x_{20}, y_{20}\right)$, vanishing at these values, and having the determinant

$$
\left|\begin{array}{llll}
g_{x_{1}} & g_{y_{i 1}} & g_{x_{2}} & g_{y_{i 2}} \\
\psi_{\rho x_{1}} & \psi_{\rho y_{i 1}} & \psi_{\rho x_{2}} & \psi_{\rho y_{i 2}}
\end{array}\right|
$$

different from zero on $E_{0}$. The new set of end conditions $\psi_{\rho}=0(\rho=1, \cdots$, $2 n+1)$ defines an auxiliary problem of Mayer of the type discussed by Bliss and Hestenes. It is clear that $E_{0}$ is also a minimizing arc for this auxiliary problem.

ThEOREM 5:1. Let $E_{0}$ be an admissible arc that is normal on the interval $x_{10} x_{20}$ and satisfies the necessary condition I. If $E_{0}$ is normal relative to the end conditions $\psi_{\mu}=0(\mu=1, \cdots, p)$, then it is normal relative to the end conditions $\psi_{\rho}=0(\rho=1, \cdots, 2 n+1)$ just defined.

To prove this theorem we recall that the matrix $(2: 4)$ has rank $p+1$ since $E_{0}$ is normal relative to the end conditions $\psi_{\mu}=0$. Furthermore since $E_{0}$ satisfies the transversality condition (2:3), it follows that on $E_{0}$ the derivatives $g_{x_{1}}, g_{y_{i 1}}, g_{x_{2}}, g_{y_{i 2}}$ are expressible as a linear combination of the rows of the matrix (2:4), the multiplier of the first row being different from zero. The rank of the matrix (2:4) formed for the new end conditions $\psi_{\rho}=0$ is therefore unaltered when the elements of the first row are replaced by the derivatives $g_{x_{1}}, g_{y_{i 1}}, g_{x_{2}}, g_{y_{i 2}}$. The matrix thus formed is the matrix of the determinant (5:1) and has rank $2 n+2$. Hence according to Theorem $2: 2, E_{0}$ is also normal relative to the end conditions $\psi_{\rho}=0$, and the theorem is established.

6. A fundamental sufficiency theorem. With the help of the auxiliary problem just constructed we can prove the following theorem:

THEOREM 6:1. A FUNDAMENTAL SUFFICIENCY THEOREM. Let $E_{0}$ be an extremal arc with the following properties:

(A). $E_{0}$ satisfies the sufficient conditions for a proper strong relative minimum 
with respect to admissible arcs $C$ satisfying the end conditions $\psi_{\rho}(C)=0$ of the auxiliary problem of Mayer defined in $\$ 5$.

(B) There is a neighborhood $M$ of the ends of $E_{0}$ in $\left(x_{1} y_{1} x_{2} y_{2}\right)$-space such that the inequality $g(E)>g\left(E_{0}\right)$ holds for every extremal $E$ of the family (3:1) with ends in $M$ satisfying the conditions $\psi_{\mu}(E)=0$ and not identical with $E_{0}$.

Then there exist neighborhoods $\mathfrak{F}$ of $E_{0}$ in xy-space and $N$ of the ends of $E_{0}$ in $\left(x_{1} y_{1} x_{2} y_{2}\right)$-space such that the inequality $g(C)>g\left(E_{0}\right)$ holds for every admissible arc $C$ in $\mathfrak{F}$ with ends in $N$ satisfying the conditions $\psi_{\mu}(C)=0$ and not identical with $E_{0}$.

The proof is based on the following two lemmas, the proofs of which will be given in the next section.

Lemma 6:1. (Modification of Hahn's Theorem (XIV, p. 129).) The property (A) for $E_{0}$ implies the existence of neighborhoods $\mathfrak{F}$ of $E_{0}$ in $x y$-space and $M$ of the ends of $E_{0}$ in $\left(x_{1} y_{1} x_{2} y_{2}\right)$-space such that for every extremal $E$ of the family (3:1) with ends in $M$ the inequality $g(C)>g(E)$ holds for every admissible arc $C$ in $\mathfrak{F}$ with ends in $M$ satisfying the conditions $\psi_{\rho}(C)=\psi_{\rho}(E)$ and not identical with $E$.

Lemma 6:2. The property (A) for $E_{0}$ implies that every neighborhood $M$ of the end values of $E_{0}$ has associated with it a second neighborhood $N$ of these end values such that for every admissible arc $C$ with ends in $N$ there is an extremal $E$ of the family (3:1) with ends in $M$ satisfying the conditions $\psi_{\rho}(C)=\psi_{\rho}(E)$.

With the help of these lemmas the proof of Theorem 6:1 is as follows. Select first neighborhoods $\mathfrak{F}$ of $E_{0}$ and $M$ of the ends of $E_{0}$ effective as in Lemma 6:1 and as in (B). Select a second neighborhood $N$ of the ends of $E_{0}$ related to $M$ as in Lemma 6:2. Consider now an admissible arc $C$ in $\mathfrak{F}$ with ends in $N$ satisfying the conditions $\psi_{\mu}=0$. According to Lemma $6: 2$ there is an extremal $E$ of the family (3:1) with ends in $M$ satisfying the conditions $\psi_{\mu}(E)=0, \psi_{\tau}(E)=\psi_{\tau}(C)$, where the functions $\psi_{\tau}$ are those adjoined to the functions $\psi_{\mu}$ to form the auxiliary Mayer problem defined in $\S 5$. From Lemma $6: 1$ it follows that $g(C) \geqq g(E)$, and from the property (B) we have $g(E)$ $\geqq g\left(E_{0}\right)$. Hence $g(C) \geqq g\left(E_{0}\right)$, the equality being valid only in case $C$ coincides with $E_{0}$, as was to be proved.

7. Proofs of two lemmas. In order to prove Lemma 6:1 we use the result obtained by Bliss and Hestenes (XVI, p. 323)* which states that the

${ }^{*}$ In the proof of the theorem referred to here, the authors made use (XVI, Theorem 8:1) of a suggestion in an abstract by Morse, Bulletin of the American Mathematical Society, vol. 37 (1931), p.37. Bliss and Reid proved Morse's result independently before the complete paper of Morse (XVII) appeared. Bliss and Hestenes used the proof given by Bliss, which is similar to that of Morse, and inadvertently made no reference to Morse's paper. The proof given by Morse should of course have priority. 
property (A) for $E_{0}$ given in Theorem 6:1 implies the existence of a function $W\left(a_{1}, \cdots, a_{n}\right)$ such that the $n$-parameter family of extremals

$$
y_{i}=y_{i}\left(x, x_{20}, a, W_{a}\right), \quad z_{i}=z_{i}\left(x, x_{20}, a, W_{a}\right) \quad\left(x_{1} \leqq x \leqq x_{2}\right)
$$

contains $E_{0}$ for parameter values $\left(x_{10}, x_{20}, a_{0}\right)$ and has the determinant $\left|y_{i a_{k}}\right|$ different from zero along $E_{0}$. Furthermore each extremal $E$ of the family (7:1) has on it the element $(x, y, z)=\left(x_{20}, a, W_{a}\right)$, where the $a_{i}$ are the parameter values defining $E$. If now we select $n-1$ functions $W_{r}\left(a_{1}, \cdots, a_{n}\right)$ having continuous first and second partial derivatives and such that the determinant $\left|W_{a_{i}} W_{r a_{i}}\right|$ is different from zero for the values $a_{i}=a_{i 0}$, then the $(2 n-1)$-parameter family of extremals

$$
\begin{aligned}
& y_{i}=y_{i}\left(x, x_{20}, a, W_{a}+b_{r} W_{r a}\right)=y_{i}(x, a, b), \\
& z_{i}=z_{i}\left(x, x_{20}, a, W_{a}+b_{r} W_{r a}\right)=z_{i}(x, a, b) \quad\left(x_{1} \leqq x \leqq x_{2}\right)
\end{aligned}
$$

contains $E_{0}$ for parameter values $\left(x_{10}, x_{20}, a_{0}, b=0\right)$. Moreover every extremal $E$ of this family has on it the element $\left(x, y_{i}, z_{i}\right)=\left(x_{20}, a_{i}, W_{a_{i}}+b_{r} W_{r a_{i}}\right)$, where the parameter values $a_{r}, b_{r}$ are those defining $E$. The equations expressing this fact are the equations

$$
a_{i}=y_{i}\left(x_{20}, a, b\right), \quad W_{a_{i}}+b_{r} W_{r a_{i}}=z_{i}\left(x_{20}, a, b\right),
$$

and by differentiation it is found that the determinant

$$
\left|\begin{array}{lll}
y_{i a_{k}} & y_{i b_{r}} & 0 \\
z_{i a_{k}} & z_{i b_{r}} & z_{i}
\end{array}\right|
$$

is different from zero for the values $(x, a, b)=\left(x_{20}, a_{0}, 0\right)$. Hence the family (7:2) is one of the type (3:1), its multipliers $\lambda_{\alpha}(x, a, b)$ being found in the usual manner (XVI, pp. 309-311).

Since the determinant $\left|y_{i a_{k}}\right|$ belonging to the family (7:1) is different from zero on $E_{0}$, the determinant $\left|y_{i a_{k}}(x, a, b)\right|$ belonging to the family $(7: 2)$ has the same property. Hence the system of equations

$$
y_{i}=y_{i}(x, a, b)
$$

has a unique solution

$$
a_{i}=a_{i}(x, y, b)
$$

in a neighborhood $\mathfrak{D}$ of the values $(x, y, b)$ belonging to $E_{0}$. The functions $a_{i}(x, y, b)$ are continuous and possess continuous derivatives of the first two orders in the domain $\mathfrak{D}$. If now we let

$$
\begin{aligned}
p_{i}(x, y, b) & =y_{i x}[x, a(x, y, b), b], \\
\lambda_{\alpha}(x, y, b) & =\lambda_{\alpha}[x, a(x, y, b), b],
\end{aligned}
$$


then according to the condition $\mathrm{II}_{\mathfrak{R}}$ ' implied by the property $(\mathrm{A})$ on $E_{0}$, the domain $\mathfrak{D}$ can be so restricted that at each element $(x, y, b)$ in $\mathfrak{D}$ the inequality

$$
E\left[x, y, p(x, y, b), \lambda(x, y, b), y^{\prime}\right]>0
$$

holds for every admissible set $\left(x, y, y^{\prime}\right) \neq(x, y, p)$, where $E\left(x, y, p, \lambda, y^{\prime}\right)$ is the Weierstrass $E$-function (XVI, pp. 317, 324). Furthermore on the hyperplane $x=x_{20}$ in $x y$-space the Hilbert integral $I^{*}$ is independent of the path when the parameters $b_{r}$ are fixed (XVI, p. 323, cf. XII, p. 269). It follows that for each set $b_{r}$ the region $\mathfrak{F}$ of points $(x, y)$, whose elements $(x, y, b)$ are all in $\mathfrak{D}$, forms a field with slope functions and multipliers defined by equations (7:4) (XVI, p. 322). We have a family of such fields depending upon the $n-1$ parameters $b_{r}$. In each field the Weierstrass $E$-function is $>0$ unless $y_{i}{ }^{\prime}=p_{i}$. Hence according to a theorem proved by Bliss and Hestenes (XVI, p. 319) there is a neighborhood $M$ of the end values of $E_{0}$ such that every extremal $E$ with ends in $M$ and belonging to one of these fields furnishes a proper strong relative minimum for the function $g$ in the class of admissible $\operatorname{arcs} C$ in $\mathfrak{F}$ whose ends are in $M$ and satisfy the conditions $\psi_{\rho}(C)=\psi_{\rho}(E)$.

Lemma 6:1 will now be established completely if we show that the neighborhood $M$ of the ends of $E_{0}$ can be restricted so that every extremal $E$ of the family $(7: 2)$ with ends in $M$ is a member of one of the fields just described. To do this we select a constant $h$ so that the set $\left[x, y, a_{i}(x, y, b), b\right]$ with elements $(x, y, b)$ in $\mathfrak{D}$ is the only solution of equations (7:3) satisfying the relation

$$
a_{i}(x, y, b)-h \leqq a_{i} \leqq a_{i}(x, y, b)+h .
$$

This can always be done since the solution $a_{i}(x, y, b)$ of equations $(7: 3)$ is isolated. We now select a constant $\epsilon$ such that the inequalities

$$
\begin{aligned}
& \left|a_{i}-a_{i 0}\right|<h / 2, \\
& \left|a_{i 0}-a_{i}(x, y, b)\right|<h / 2
\end{aligned}
$$

hold along every extremal $E$ of the family $(7: 2)$ with parameter values $\left(x_{1}\right.$, $\left.x_{2}, a, b\right)$ in an $\epsilon$-neighborhood of those belonging to $E_{0}$. The relation $(7: 5)$ now holds for every set of values $(x, y, a, b)$ on $E$. It follows that $a_{i}=a_{i}(x, y, b)$, and hence $E$ is an extremal of one of the fields just described. This completes the proof of Lemma 6:1 since according to Theorem 3:1 the neighborhood $M$ of the ends of $E_{0}$ can be so restricted that every extremal $E$ of the family $(7: 2)$ with ends in $M$ has parameter values $\left(x_{1}, x_{2}, a, b\right)$ in the $\epsilon$-neighborhood just defined. 
In order to prove Lemma $6: 2$ consider first the equations

$$
\begin{aligned}
& W\left(x_{1}, y_{1}, x_{2}, y_{2}\right)=0, \\
& \psi_{\rho}\left(x_{1}, y_{1}, x_{2}, y_{2}\right)=m_{\rho},
\end{aligned}
$$

where $W$ is the function defined in Theorem $3: 1$. As was seen in $\S 3$, the functional determinant of these equations is different from zero on $E_{0}$. Furthermore equations $(7: 6)$ are satisfied by the set $\left(x_{1}, y_{1}, x_{2}, y_{2}, m\right)=\left(x_{10}, y_{10}, x_{20}\right.$, $\left.y_{20}, 0\right)$ belonging to $E_{0}$. Hence there is a constant $h>0$ such that equations $(7: 6)$ have a unique solution

$$
\begin{array}{ll}
x_{1}=x_{1}(m), & y_{i 1}=y_{i 1}(m), \\
x_{2}=x_{2}(m), & y_{i 2}=y_{i 2}(m)
\end{array}
$$

for all values $m_{\rho}$ satisfying the relations $\left|m_{\rho}\right|<h$. If $h$ is sufficiently small, then according to Theorem 3:1 every pair of points $\left(x_{1}, y_{1}\right),\left(x_{2}, y_{2}\right)$ can be joined by an extremal of the family $(3: 1)$. Furthermore it is clear that, if necessary, the constant $h$ can be further restricted so that every set of values $\left(x_{1}, y_{1}, x_{2}, y_{2}\right)$ defined by equations (7:7) with $\left|m_{\rho}\right|<h$ is in a preassigned neighborhood $M$ of the end values of $E_{0}$. If now we select a second neighborhood $N$ of the end values of $E_{0}$ so that every set of values $\left(x_{1}, y_{1}, x_{2}, y_{2}\right)$ in $N$ satisfies the relation $\left|\psi_{\rho}\left(x_{1}, y_{1}, x_{2}, y_{2}\right)\right|<h$, then every admissible arc $C$ with ends in $N$ determines a set of values $m_{\rho}=\psi_{\rho}(C)$ satisfying the relation $\left|m_{\rho}\right|$ $<h$, and these in turn determine an extremal arc $E$ with ends in $M$ satisfying the conditions $\psi_{\rho}(E)=\psi_{\rho}(C)$. This proves Lemma 6:2.

8. Sufficient conditions for relative minima. The necessary condition $I$ is given in $\$ 2$. The symbols II $_{\mathfrak{R}}$, III' will be used to denote the strengthened conditions of Weierstrass and Clebsch as defined by Bliss and Hestenes (XVI, p. 324). The symbol IV' will be used to denote the condition IV of $\$ 4$ strengthened so as to exclude the equality sign. With these definitions agreed upon we can state the following theorem:

THEOREM 8:1. SUFFICIENT CONDITIONS FOR A STRONG RELATIVE MINIMUM. Let $E_{0}$ be an admissible arc without corners and with end points determined by values $x_{10}, x_{20}$ and satisfying the conditions $\psi_{\mu}=0$. If $E_{0}$ is normal relative to the end conditions $\psi_{\mu}=0$, is normal on every sub-interval $x_{10} x_{3}$ of $x_{10} x_{20}$, and satisfies the conditions I, II ', III', IV', then there exist neighborhoods $\mathfrak{F}$ of $E_{0}$ in $x y$ space and $N$ of the ends of $E_{0}$ in $\left(x_{1} y_{1} x_{2} y_{2}\right)$-space such that the inequality $g(C)$ $>g\left(E_{0}\right)$ holds for every admissible arc $C$ in $\mathfrak{F}$ with ends in $N$ satisfying the conditions $\psi_{\mu}(C)=0$ and not identical with $E_{0}$.

The theorem will be established if we can show that the hypotheses of the 
theorem imply those of Theorem 6:1. It is easily seen from Theorem 5:1 and from the sufficiency conditions given by Bliss and Hestenes for the case $p=2 n+1$ (XVI, p. 324) that $E_{0}$ is an extremal arc having the property (A) of Theorem 6:1 provided that we can show that the condition IV', as defined above, implies that the ends of $E_{0}$ are not conjugate to each other. If the ends of $E_{0}$ were conjugate then the constants $d c_{s}$ in the expressions $\delta y_{i}=y_{i c_{s}} d c_{s}$ could be selected not all zero so that the differentials $\delta y_{i}$ would all vanish at the ends of $E_{0}$. If we should take these constants $d c_{s}$ together with the values $d x_{1}=d x_{2}=0$, then the conditions $d \psi_{\mu}=0$ would be satisfied and the expression $(4: 2)$ for $d^{2} g$ would vanish, which would contradict the condition IV'. Hence $E_{0}$ has property (A) of Theorem 6:1.

To prove that $E_{0}$ has the property (B) of Theorem 6:1 we first note that the conditions I, III' imply the existence of a family of extremals (3:1) containing $E_{0}$ for parameter values $\left(x_{10}, x_{20}, c_{s 0}\right)$. From conditions I, IV' it follows that $d g=0, d^{2} g>0$ for every set of differentials $\left(d x_{1}, d x_{2}, d c_{s}\right) \neq(0,0,0)$ which satisfy the conditions $d \psi_{\mu}=0$. But these are the conditions (XV, p. 115) which insure that $g\left(x_{1}, x_{2}, c_{s}\right)>g\left(x_{10}, x_{20}, c_{s 0}\right)$ for all sets $\left(x_{1}, x_{2}, c_{s}\right) \neq\left(x_{10}, x_{20}, c_{s 0}\right)$ satisfying the equations $\psi_{\mu}\left(x_{1}, x_{2}, c_{s}\right)=0$ and lying in a sufficiently small $\epsilon$-neighborhood of $\left(x_{10}, x_{20}, c_{s 0}\right)$. Furthermore since the ends of $E_{0}$ are not conjugate the matrix (3:2) has rank $2 n-1$ (XVI, p. 316), and according to Theorem 3:1 there is a neighborhood $M$ of the ends of $E_{0}$ such that every extremal with ends in $M$ has parameter values $\left(x_{1}, x_{2}, c_{s}\right)$ in the $\epsilon$-neighborhood described above. It follows that $g\left(x_{1}, y_{1}, x_{2}, y_{2}\right)>g\left(x_{10}, y_{10}, x_{20}, y_{20}\right)$ for every extremal $E$ with ends in $M$ satisfying the conditions $\psi_{\mu}(E)=0$ and not identical with $E_{0}$. Hence $E_{0}$ has the property (B) of Theorem 6:1 and Theorem $8: 1$ is established.

In a similar manner sufficient conditions for a weak relative minimum for the general problem of Mayer with variable end points can be established. The argument is like that of Bliss and Hestenes (XVI, p. 325) with the help of simple modifications of Theorem 6:1 and Lemma 6:1 above. The Theorem $10: 2$ of Bliss and Hestenes remains valid here if we replace the phrase "preceding theorem" by "Theorem 8:1" and the equations $\psi_{\rho}=0$ by $\psi_{\mu}=0$. Similarly Corollary 10:1 of the paper by Bliss and Hestenes is still effective if we replace "Theorem 10:1" by "Theorem 8:1" and $\psi_{\rho}$ by $\psi_{\mu}$.

\section{BIBLIOGRAPHY}

The papers listed below are a continuation of the list at the end of the paper of Bliss and Hestenes cited here as XVI.

XIII. Mayer, Zur A ufstellung der Kriterion des Maximums und Minimums der einfachen Integrale bei variablen Grenzwerten, Leipziger Berichte, vol. 36 (1884), pp. 99-128, vol. 48 (1896), pp. 436-465. 
XIV. Hahn, Ueber Variationsprobleme mit variablen Endpunkten, Monatshefte für Mathematik und Physik, vol. 22 (1911), pp. 127-136.

XV. Hancock, Theory of Maxima and Minima, Ginn and Company, 1917.

XVI. Bliss and Hestenes, Sufficient conditions for a problem of Mayer in the calculus of variations, these Transactions, vol. 35 (1933), pp. 305-326.

XVII. Morse, Sufficient conditions in the problem of Lagrange with fixed end points, Annals of Mathematics, (2), vol. 32 (1931), pp. 567-577.

University of. Chicago,

Chicago, Inl. 